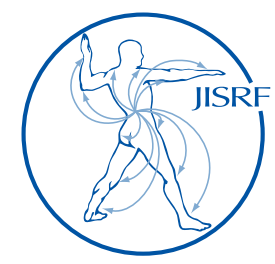

Check for updates

\title{
Multimodal Venous Thromboembolism Prophylaxis with Preoperative Thrombophilia Screening Examinations for Total Hip and Knee Arthroplasties
}

Oshima $Y^{1}$, Fetto $\mathrm{J}^{1}$

\section{Abstract}

Objective: To evaluate the efficacy of a multimodal venous thromboembolism (VTE) prophylaxis including preoperative thrombophilia screening for total hip and knee arthroplasties (THAs and TKAs) and to consider the possibility of utilizing thrombophilic blood markers for preoperative identification of patients with high risk for VTE.

Method: The physical evaluation, involving the medical history of previous VTE, recent malignancy, and preoperative prolonged immobility status, and the existence of deep venous thrombosis (DVT) detected by duplex venous ultrasonography were assessed. Then, the patients with high risk of VTE were offered an inferior vena cava (IVC) filter preoperatively. The laboratory examination of complete blood count and standard biochemistry with factor VIII, activated protein C resistance (APCR), and prothrombin gene mutation were also measured. The operations were performed under regional anesthesia in most cases, and with venous foot pump (VFP), and early mobilization and aspirin (325 mg) daily were applied postoperatively.

Results: IVC filters were placed in 6, and acute DVT was detected in 5 of the total 99 cases. However, there was no critical bleeding or fatal VTE. In the blood markers, prothrombin mutation and factor VIII seemed to have a relation to DVT.

Conclusion: The efficacy of our multimodal protocol was confirmed. Further research is necessary to apply factor VIII and prothrombin gene mutation as thrombophilic blood markers.

Keywords: total hip and knee arthroplasties, multimodal venous thromboembolism prophylaxis, thrombophilia screening examinations, factor VIII, prothrombin gene mutation

Level of Evidence: AAOS Therapeutic Level IV

1 Yasushi Oshima, MD, PhD; Joseph Fetto, MD

Division of Adult Reconstructive Surgery, Department of Orthopaedic Surgery, New York University Hospital for Joint Diseases, 1040 First Avenue, \#345, New York, NY 10022 USA (Direct reprint requests to Yasushi Oshima)

\author{
(c) 2015 Oshima, Fetto. All rights reserved \\ Authors retain copyright and grant the journal right of first publication with the work. \\ Reconstructive Review follows the Creative Commons Attribution-NonCommercial \\ CC BY-NC. This license allows anyone to download works, build upon the material, \\ and share them with others for non-commercial purposes as long as they credit the \\ senior author, Reconstructive Review, and the Joint Implant Surgery \& Research \\ Foundation (JISRF). An example credit would be: "Courtesy of (senior author's name), \\ Reconstructive Review, JISRF, Chagrin Falls, Ohio".
}


Abbreviations: total hip and knee arthroplasties (THAs and TKAs); venous thromboembolism (VTE); deep venous thrombosis (DVT); pulmonary embolism (PE); inferior vena cava (IVC); intermittent pneumatic compression (IPC); venous foot pump (VFP); low molecular weight heparin (LMWH); activated protein C resistance (APCR)

\section{Introduction}

Total hip and knee arthroplasties (THAs and TKAs) are highly successful treatments for severe arthritis to remove pain, increase functional ability, and improve the quality of life. [1] In 2009, there were an estimated 284,000 primary THAs, 45,000 revision THAs, 619,000 primary TKAs, and 59,500 revisions TKAs performed in the USA, and these numbers are expected to increase in the future. [2]

Generally, the factors related to surgery including the operative procedures, the use of pneumatic tourniquet, and the heat of bone cement, together with the patients' inherent factors and intra- and post-operative immobilization are thrombogenic stimuli, which are part of Virchow's triad of hypercoagulability, hemodynamic stasis, and endothelial injury. [3] The surgery is a traumatic event that increases the risk of venous thromboembolism (VTE) including deep venous thrombosis (DVT) and pulmonary embolism (PE). Especially THAs and TKAs have been reported to have high incidence of VTE in surgery. [4, 5]

The incidence of DVT without prophylaxis after THAs and TKAs has been reported to be 42 to $57 \%$ and 41 to $85 \%$, and the incidence of fatal PE is 0.1 to $2.0 \%$ and 0.1 to $1.7 \%$, respectively. [6] However, VTE is considered to be a preventable cause of hospital readmission and death. $[7,8]$

Traditional VTE prophylaxis is composed of mechanical and pharmacological categories. The mechanical treatments with early mobilization, the use of elastic stocking, intermittent pneumatic compression (IPC) and venous foot pump (VFP) devices work against the immobility status to prevent blood stagnation and promote blood circulation. [9] The pharmacological prophylaxis has been developed with many anticoagulant agents, i.e. aspirin, warfarin, unfractionated heparin, low molecular weight heparin (LMWH), factor Xa inhibitors, and thrombin inhibitors. However, the most effective or the gold standard treatment for VTE prophylaxis with the optimum outcome, minimum risk and acceptable expense after THAs and TKAs remains undecided. [10] This situation has been made more confusing by the assumption discussed by all of the published studies, that all patients undergoing THAs and TKAs present with an equal genetic potential for suffering of a VTE event. This is a wholly unsupported assumption which compromises the conclusions made regarding the benefits of one prophylaxis protocol or agent over another.

A multimodal thromboprophylaxis with regional anesthesia, VFP, early mobilization and aspirin has been applied at our institution, and the outcome was acceptable without any critical complications or fatal VTE. [11,12] However, the question remains of whether additional approaches are needed for preventively and objectively identifying patients with inherent or acquired thrombophilia condition. [13]

We hypothesized that a multimodal VTE prophylaxis with aspirin would be able to perform even better if we could identify and exclude patients with a demonstrated increased risk for VTE. Thus, the primary objective of the present study was to evaluate the efficacy of a multimodal VTE prophylaxis with preoperative thrombophilia screening examinations involving preoperative physical evaluations and duplex venous ultrasonography for THAs and TKAs. Moreover, many blood markers for thrombophilia screening have been reported, however, it is still debatable which markers are reliable and how to use these markers for patient selection before surgery. Therefore, the second objective was to consider the possibility of utilizing thrombophilic blood markers for preoperative identification of patients with high risk for VTE.

\section{Patients and Methods}

Between January 2011 and June 2014, 135 primary and revision THAs and TKAs were performed by a single surgeon with a multimodal VTE prophylaxis in 113 unselected, consecutive patients. Prior to the surgery, all patients received preoperative physical evaluations by their primary care physicians. Patients without all items of laboratory examinations were excluded.

\section{Preoperation}

To detect thrombophilia as a high risk condition for VTE, physical evaluation, involving the medical history of previous VTE, recent malignancy, and preoperative prolonged immobility status, and the existence of DVT by duplex venous ultrasonography were assessed for all pre-surgical patients. Moreover, laboratory examination of complete blood count and standard biochemistry with factor VIII, activated protein $\mathrm{C}$ resistance (APCR), and prothrombin gene mutation were measured.

Those patients with positive physical evaluations and a pre-existing DVT were regarded as having an increased thromboembolic risk. These patients were examined by hematologists at our institute, and treatments with a removable inferior vena cava 
(IVC) filter and anticoagulants of warfarin, heparin, or LMWH, were applied to maintain blood coagulation level at international normalized ratio of 2.0, when necessary. IVC filters were usually removed 3 months after surgery.

\section{Intraoperation}

Regional anesthesia was undertaken, in which the physical conditions were stable and pain management was achieved. Otherwise, patients were operated under general anesthesia.

THAs were performed through a posterior approach with noncemented FMP Acetabular and Revelation Hip System (DJO Global, Vista, CA). TKAs were performed through a parapatellar approach with cemented 3DKnee System (DJO Global, Vista, CA). Patella was replaced in all cases. Pneumatic tourniquet was applied only during cementing process, usually 15 to 20 minutes.

VFP (Covidien, Mansfield, MA) were worn on the contralateral site foot from the beginning of surgery, and on the surgical site from just after surgery. This device consists of an anatomically shaped inflation pad and cushioned foot cover that can withstand a period of rapid inflation ( 0.4 seconds) followed by a 3 second impulse hold time to achieve a pressure of $130 \mathrm{mmHg}$ and a 20 second deflation period.

\section{Postoperation}

VFP were worn on both feet throughout hospitalization, usually for two to three days after surgery. Ankle and toe mobilization was started with a physical therapist from the night of surgery. Full weight bearing ambulation with a walker, crutches, or a cane was started from the morning of the first postoperative day. Patients received two sessions of physical therapy per day during their hospitalization. Aspirin (acetylsalicylic acid) $325 \mathrm{mg}$ daily was administrated for six weeks, started from the night of surgery. Celecoxib (COX-2 selective nonsteroidal anti-inflammatory drug) $400 \mathrm{mg}$ per day and oral opioids were prescribed for breakthrough pain.

All patients were followed for three months after surgery, and none was lost to follow-up. Standardized clinical criteria were used to identify probable cases of DVT (tenderness or discomfort of the calf or thigh, a positive Homan's sign, edema of the leg and ankle, and increase in local temperature) or PE (pleuritic chest pain, dyspnea, arterial blood gas measurements, changes in the chest radiograph and/ or changes in the electrocardiogram). [11] Routine venography or duplex venous ultrasonography was not performed.
This study was approved by Institution Review Board (IRB). All patients provided informed consent. No funding or financial benefits were provided to the authors for the project from any source.

\section{Statistical Analysis}

Descriptive statistics were used to describe the subject population and evaluate the incidence of VTE. Fisher's exact tests were used to compare the rates of VTE for each of the variables included. Logistic regression was used to identify factors related to the occurrence of VTE while controlling for all other variables. All statistical analyses were performed with SPSS version 19.0 software (SPSS Inc., Chicago, Il), and significance level was set at $p<0.05$.

\section{Results}

Ninety-nine cases in 83 patients were enrolled. Sixteen patients had received 2 separated surgeries during this time periods (Table 1). Of 99 cases, 42 were male and 57 were female. The mean age was 68 years (range from 36 to 92 years). Fifty-two cases were primary THAs, 32 were primary TKAs, 11 were revision THAs, and 4 were revision TKAs. Six patients with 8 cases were severely obese (40 $\mathrm{kg} / \mathrm{m}^{2}>\mathrm{BMI} \geq 35 \mathrm{~kg} / \mathrm{m}^{2}$ ), and 5 patients with 6 cases were morbidly obese (BMI $\geq 40 \mathrm{~kg} / \mathrm{m}^{2}$ ). The procedures of anesthesia were regional in 81 cases (81.8\%) (femoral nerve block : 1 , epidural : 21 , spinal : 59), and general in 18 cases $(18.2 \%)$.

Table 1: Patients with 2 surgeries

\begin{tabular}{|l|l|}
\hline Total number of patients: & 16 \\
\hline Simultaneous bilateral THA: & 5 \\
\hline Simultaneous bilateral TKA: & 6 \\
\hline Two staged bilateral THA: & 1 \\
\hline Two staged bilateral TKA: & 1 \\
\hline Primary THA and revision THA: & 2 \\
\hline $\begin{array}{l}\text { Revision THA and contralateral } \\
\text { primary THA: }\end{array}$ & 1 \\
\hline
\end{tabular}

One patient with a revision THA had already had a permanent IVC filter in place at the time of initial consultation, because of a prior history of PE and chronic distal DVT. No additional VTE treatment was performed for this patient, however, he did not have any further episode of VTE. IVC filters were placed for 6 cases in 5 additional patients, and thus, the total was 7 cases in 6 patients. 
Acute DVT was detected postoperatively in 5 cases in 4 patients, all of which were distal. An IVC filter had been newly placed preoperatively in only one among these 5 cases in 4 patients with acute DVT, and this was the only case of acute DVT among the 6 newly placed filter cases.

Six patients had previous VTE histories, and 1 patient, as mentioned above, had already received a filter. Because 2 out of 5 patients had rejected treatments with a filter and additional anticoagulants, these patients received only the standard treatment with aspirin, and the other 3 patients received new filters and additional anticoagulant treatments. One patient with a newly placed filter had acute DVT after surgery, however, there was no fatal VTE.

All patients suffered from severe hip or knee pain preoperatively, however, none were categorized as critically immobilized.

For malignancy, 6 patients with 8 cases had histories, and 5 patients with 6 cases were in remission. One patient with 2 cases was suffering from an advanced stage breast cancer, however, that patient rejected any additional anticoagulant and/or filter treatment. She received only our standard treatment, however, there was no critical VTE after surgery. Thus, no filter nor additional anticoagulant treatment was applied for malignancy in any cases.

Ten patients with 15 cases had histories of cardiovascular diseases, and they consulted cardiologists. Most of these were treated with warfarin, started before surgery.

In the preoperative laboratory examinations, factor VIII was elevated in 40 patients with 49 cases, in which all the acute and chronic DVT cases were included $(\mathrm{P}=0.012)$. however, these cases did not involve any VTE episode. Heterozygosity of prothrombin gene was shown in 4 patients with 5 cases, in which one case had a prior VTE history, 1 case had prior VTE and chronic DVT, and 1 case had prior VTE and acute DVT (Table 2).

A 71-year-old male, who had suffered from hypertension, hypertrophic obstructive cardiomyopathy, aortic regurgitation, and diabetes, died 2 weeks after primary TKA under general anesthesia. However, autopsy determined APCR was lower than normal in 2 patients with 2 cases,

cause of death to be acute myocardial infarction. Consequently, there was no patient readmitted or reoperated with critical bleeding, major wound complications, or critical VTE.

\section{Discussion}

VTE is a common multicausal disease affected by interaction of genetic, environmental and behavioral factors. [14] Many surgeons tend to focus on DVT prophylaxis to prevent VTE, however, low correlation has been found between DVT and PE. [9,15] Accordingly, we have been applying a multimodal VTE prophylaxis to manage each risk factor of VTE step by step.

Firstly, to minimize the immobilization period, VFP and early mobilization are applied. However, additional chemoprophylaxis is still thought to be necessary, especially for patients at high risk for VTE. [9]

Recently, many anticoagulant agents have been developed and applied to target different steps of the coagulation cascade, in which fibrin becomes blood clot at the end. The balancing of clotting and bleeding is essential, however, some of the recent anticoagulants are costly and may increase such wound complications as hematoma, delayed wound healing, surgical site infection, and uncontrolled bleeding. $[13,15]$ Thus, the routine use of these agents for all patients has been questioned. [16]

Aspirin, an antiplatelet drug, does not directly affect the coagulation cascade, and thus results in much less bleeding complication than aggressive anticoagulants. [15] Recently, the two most commonly applied guidelines, published by the American College of Chest Physicians (ACCP) and the American Academy of Orthopaedic Surgeons (AAOS) in the USA, have accepted aspirin as a VTE prophylaxis agent. [17]

The use of regional anesthesia has been shown to decrease the incidence of VTE and to attain the rehabilitative goals more rapidly compared to general anesthesia. [18] Consequently, regional anesthesia was applied for most of our cases, other than the physically morbid and Table 2: Heterozygosity of prothrombin gene mutation

\begin{tabular}{|l|l|l|l|l|l|l|l|}
\hline Age, Gender & Factor VII & APCR & Surgery & Anesthesia & VTE History & Filter & DVT \\
\hline $71-\mathrm{y}-\mathrm{o}$, male & 219 & 2.9 & $\begin{array}{l}\text { Revision } \\
\text { THA }\end{array}$ & General & $\begin{array}{l}\text { PE and chronic } \\
\text { DVT }\end{array}$ & Prior filter & Chronic \\
\hline $\begin{array}{l}70-\mathrm{y}-\mathrm{o}, \\
\text { female }\end{array}$ & 207 & 3.05 & $\begin{array}{l}\text { Revision } \\
\text { THA }\end{array}$ & Regional & None & $\begin{array}{l}\text { Received a } \\
\text { new filter }\end{array}$ & Acute \\
\hline $85-\mathrm{y}-\mathrm{o}$, male & 141 & 2.84 & $\begin{array}{l}\text { Revision } \\
\text { THA }\end{array}$ & Regional & DVT & $\begin{array}{l}\text { Received a } \\
\text { new filter }\end{array}$ & None \\
\hline 91-y-0, male & 106 & 2.59 & $\begin{array}{l}\text { Two staged } \\
\text { TKAs }\end{array}$ & Regional & None & No filter & None \\
\hline
\end{tabular}
some bilateral and revision cases. Because of its low risk for bleeding complication, aspirin could be applied safely even after spinal and epidural anesthesia. [15]

The efficacy and safety of VTE pro- 
phylaxis using regional anesthesia, VFP, early mobilization and aspirin for THAs and TKAs had been confirmed. $[4,11,12,15,16,19]$ However, it is still debatable whether aspirin is adequate for high risk patients of VTE. [13] Thus, preoperative screening examinations were used to detect and eliminate high risk patients, rather than applying the potent anticoagulants routinely to all. Obesity is commonly considered to be a risk factor of VTE. [4,16] However, while obesity has been reported to be a risk for peripheral edema, wound inflammation, and wound infection, $[20,21]$ at least, no critical VTE was detected in the severe and morbid obese patients in the present study. Cardiovascular disease is a high risk for arterial complications, and actually one patient in this study died of acute myocardial infarction. However, cardiovascular disease, itself, is not directly linked to high risk of VTE. [14]

Blood markers have also been examined to detect thrombophilia conditions. [14,22] D-dimer rises during an acute event of VTE, however, it has been reported not to be an adequate marker for VTE risk screening, because the value easily varies depending upon the conditions and timing of taking blood samples. [23]

Elevated factor VIII has been reported to increase the risk of VTE. [22,23] Forty-nine among our 99 cases $(49.5 \%)$, i.e. 40 among 83 patients showed elevated factor VIII levels, and all acute and chronic DVT cases in our study were included among this group with statistical significance. However, further research for the threshold of this marker for VTE risk screening is necessary. Factor V Leiden is a common genetic risk factor for VTE, and over $80 \%$ of the APCR phenotype can be explained by the factor V Leiden mutation. [24] Thus, we examined APCR levels, and 2 patients with 2 cases showed lower than normal, however, they had no episode of VTE before or after surgery. Prothrombin gene mutation has been shown to be associated with a high risk of VTE. [24] In the present study, prothrombin mutation seemed to have a relation to VTE. However, the benefit and accuracy of routine blood marker examinations for thrombophilia screening could not be proven even in our results. [24-26] Thus, blood markers have been used only as a reference at our institute.

After preoperative thrombophilia screening, we placed new filters and administered additional anticoagulants for 5 patients, and one among those 5 patients developed acute DVT. The remaining 4 patients did not have any VTE episodes after surgery. However, we do not know whether these were over treated or if VTE had actually been prevented in some patients by the multimodal prophylaxis.

This study has several limitations. It was a retrospective analysis based on a data source. It was not designed to investigate the incidence of VTE, nor did it include a control group. DVT evaluation was performed only for symptomatic patients, and thus asymptomatic DVT was not elucidated. Although we had never had any case of critical VTE with our multimodal VTE prophylaxis before introducing the VTE risk screening, we thought the screening to be beneficial for detecting high risk patients preoperatively. $[11,12]$ Therefore, we applied the preoperative thrombophilia screening examinations, and tried to evaluate their usefulness in this study. Unfortunately, many patients had to be excluded from this study because of lack of blood marker data from primary care physicians.

The costs of blood screening examinations, duplex venous ultrasonography evaluation and IVC filter placement are much less than those associated with the use of recent anticoagulants or the cost for readmission, reoperation, or critical PE treatment. Therefore, our multimodal prophylaxis with preoperative thrombophilia screening is thought to be beneficial for THAs and TKAs, as it reduces the need for aggressive anticoagulants.

\section{Conclusions}

As there was neither critical bleeding nor fatal VTE when applied for THAs and TKAs, the efficacy of our multimodal VTE prophylaxis with preoperative thrombophilia screening examinations and mechanical and pharmacological prophylaxis has been confirmed. Further research, however, is necessary to discuss factor VIII and prothrombin gene mutation as useful markers for preoperative VTE risk assessment.

\section{References}

1. Ethgen $\mathrm{O}$, Bruyère $\mathrm{O}$, Richy $\mathrm{F}$, Dardennes $\mathrm{C}$, Reginster JY. Health-related quality of life in total hip and total knee arthroplasty. A qualitative and systematic review of the literature. J Bone Joint Surg Am. 2004 May;86-A(5):963-74. [PubMed]

2. Kurtz SM, Ong KL, Lau E, Bozic KJ. Impact of the economic downturn on total joint replacement demand in the United States: updated projections to 2021. J Bone Joint Surg Am. 2014 Apr 16;96(8):624-30. [PubMed] [CrossRef]

3. Lopez JA, Chen J. Pathophysiology of venous thrombosis. Thromb Res 2009, 123(Suppl 4):S30-34. [PubMed] [CrossRef]

4. Vulcano E, Gesell M, Esposito A, Ma Y, Memtsoudis SG, Gonzalez Della Valle A. Aspirin for elective hip and knee arthroplasty: a multimodal thromboprophylaxis protocol. Int Orthop. 2012 Oct;36(10):1995-2002. [PubMed] [CrossRef]

5. Yassin M, Mitchell C, Diab M, Senior C. The necessity of pharmacological prophylaxis against venous thromboembolism in major joint arthroplasty. Int Orthop. 2014 May;38(5):1073-5. [PubMed] [CrossRef]

6. Geerts WH, Pineo GF, Heit JA, Bergqvist D, Lassen MR, Colwell CW, Ray JG. Prevention of venous thromboembolism: the Seventh ACCP Conference on Antithrombotic and Thrombolytic Therapy. Chest. 2004 Sep;126(3 Suppl):338S-400S [PubMed] [CrossRef]

7. Drescher FS, Sirovich BE, Lee A, Morrison DH, Chiang WH, Larson RJ. Aspirin versus anticoagulation for prevention of venous thromboembolism major lower extremity orthopedic surgery: a systematic review and meta-analysis. J Hosp Med. 2014 Sep;9(9):579-85. [PubMed] [CrossRef]

8. Lindblad B, Eriksson A, Bergqvist D. Autopsy-verified pulmonary embolism in 
a surgical department: analysis of the period from 1951 to 1988. Br J Surg. 1991 Jul;78(7):849-52. [PubMed] [CrossRef]

9. Hamilton WG, Reeves JD, Fricka KB, Goyal N, Engh GA, Parks NL. Mechanical Thromboembolic Prophylaxis With Risk Stratification in Total Knee Arthroplasty. J Arthroplasty. 2015 Jan;30(1):43-5. [ [ PubMed] [CrossRef]

10. Eikelboom JW, Karthikeyan G, Fagel N, Hirsh J. American Association of Orthopedic Surgeons and American College of Chest Physicians guidelines for venous thromboembolism prevention in hip and knee arthroplasty differ: what are the implications for clinicians and patients? Chest. 2009 Feb;135(2):513-20. [PubMed] [CrossRef]

11. Leali A, Fetto FJ, Moroz A. Prevention of thromboembolic disease after noncemented hip arthroplasty. A multimodal approach. Acta Orthop Belg. 2002 Apr;68(2):128-34. [PubMed]

12. Ragucci MV, Leali A, Moroz A, Fetto FJ. Comprehensive deep venous thrombosis prevention strategy after total-knee arthroplasty. Am J Phys Med Rehabil. 2003 Mar;82(3):164-8. [PubMed]

13. Budhiparama NC, Abdel MP, Ifran NN, Parratte S. Venous Thromboembolism (VTE) Prophylaxis for Hip and Knee Arthroplasty: Changing Trends. Curr Rev Musculoskelet Med. 2014 Jun;7(2):108-16. [PubMed] [CrossRef]

14. Rosendaal FR. Venous thrombosis: the role of genes, environment, and behavior. Hematology Am Soc Hematol Educ Program. 2005:1-12. [PubMed] [CrossRef]

15. Lotke PA, Lonner JH. The benefit of aspirin chemoprophylaxis for thromboembolism after total knee arthroplasty. Clin Orthop Relat Res. 2006 Nov;452:17580. [PubMed]

16. Gesell MW, González Della Valle A, Bartolomé García S, Memtsoudis SG, Ma Y, Haas SB, Salvati EA. Safety and efficacy of multimodal thromboprophylaxis following total knee arthroplasty: a comparative study of preferential aspirin vs. routine coumadin chemoprophylaxis. J Arthroplasty. 2013 Apr;28(4):575-9. [PubMed] [CrossRef]

17. Stewart DW, Freshour JE. Aspirin for the prophylaxis of venous thromboembolic events in orthopedic surgery patients: a comparison of the AAOS and ACCP guidelines with review of the evidence. Ann Pharmacother. 2013 Jan;47(1):63-74. [PubMed] [CrossRef]
18. Rodgers A, Walker N, Schug S, McKee A, Kehlet H, van Zundert A, Sage D, Futter M, Saville G, Clark T, MacMahon S. Reduction of postoperative mortality and morbidity with epidural or spinal anaesthesia: results from overview of randomised trials. BMJ. 2000 Dec 16;321(7275):1493-1505. [PubMed] [CrossRef]

19. Salvati EA, Sharrock NE, Westrich G, et al. The 2007 ABJS Nicolas Andry Award: three decades of clinical, basic, and applied research on thromboembolic disease after THA: rationale and clinical results of a multimodal prophylaxis protocol. Clin Orthop Relat Res 2007;459:246. [PubMed]

20. White RH, Henderson MC. Risk factors for venous thromboembolism after total hip and knee replacement surgery. Curr Opin Pulm Med. 2002 Sep;8(5):365-71. [PubMed]

21. Friedman RJ, Hess S, Berkowitz SD, Homering M. Complication rates after hip or knee arthroplasty in morbidly obese patients. Clin Orthop Relat Res. 2013 Oct;471(10):3358-66. [PubMed] [CrossRef]

22. Jenkins PV, Rawley O, Smith OP, O'Donnell JS. Elevated factor VIII levels and risk of venous thrombosis. Br J Haematol. 2012 Jun;157(6):653-63. [PubMed] [CrossRef]

23. Pabinger I, Ay C. Biomarkers and venous thromboembolism. Arterioscler Thromb Vasc Biol. 2009 Mar;29(3):332-6. [PubMed] [CrossRef]

24. Wåhlander K, Larson G, Lindahl TL, Andersson C, Frison L, Gustafsson D, Bylock A, Eriksson BI. Factor V Leiden (G1691A) and prothrombin gene G20210A mutations as potential risk factors for venous thromboembolism after total hip or total knee replacement surgery. Thromb Haemost. 2002 Apr;87(4):580-5. [PubMed]

25. Ho WK, Hankey GJ, Eikelboom JW. Should adult patients be routinely tested for heritable thrombophilia after an episode of venous thromboembolism? Med J Aust. 2011 Aug 1;195(3):139-42. [PubMed]

26. Hornsby LB, Armstrong EM, Bellone JM, Treadway S, Phillippe HM. Thrombophilia Screening. J Pharm Pract. 2014 Apr 16;27(3):253-259. [PubMed] [Cross$\underline{\text { Ref] }}$

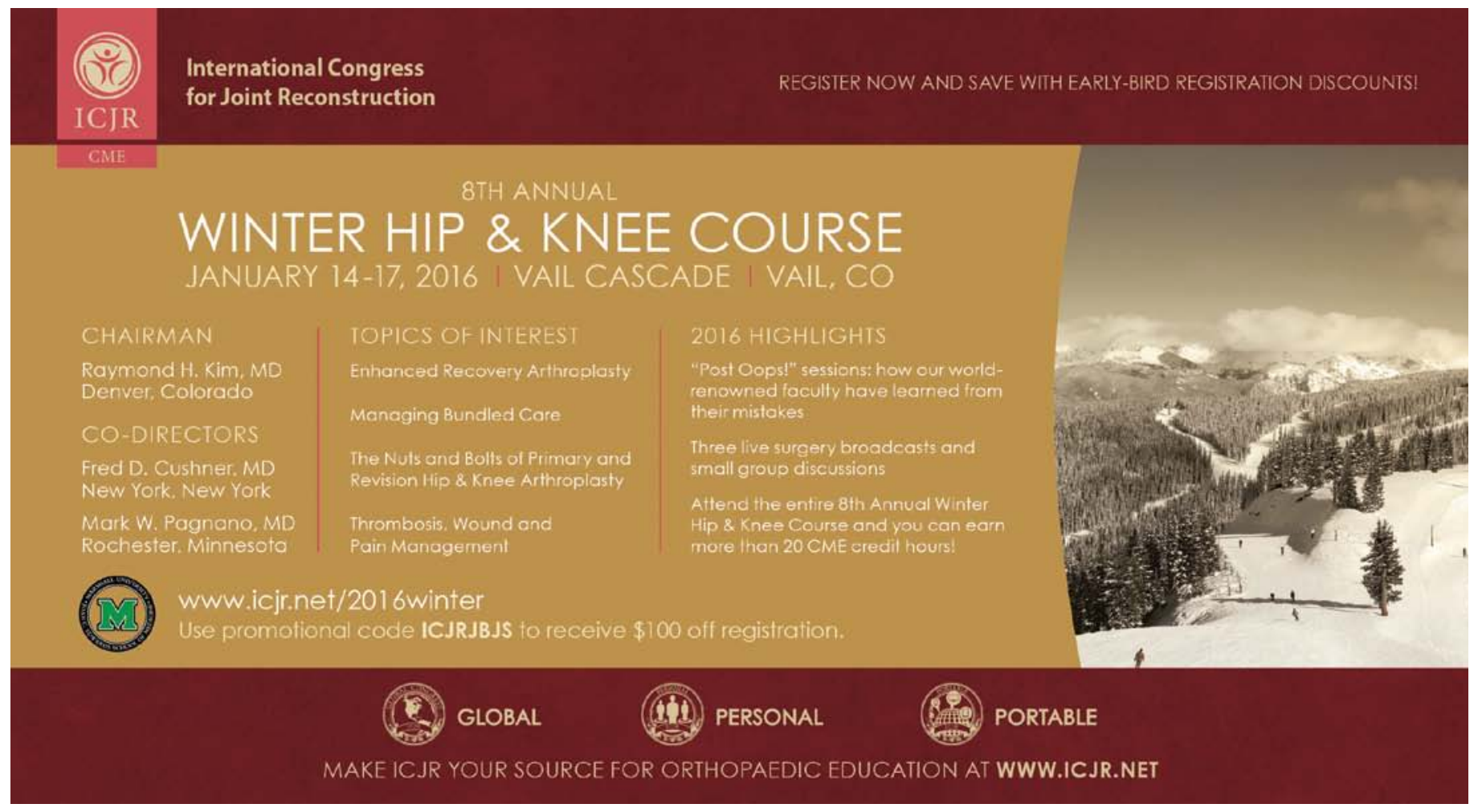

Joint Implant Surgery \& Research Foundation • JISRF.org • ReconstructiveReview.org 